\section{Achados clínicos e endoscópicos em crianças com estridor}

\author{
José F. Lubianca Neto', Gilberto B. Fischer' ${ }^{2}$, \\ Fernanda D. Peduzzi ${ }^{3}$, Humberto L. Junior ${ }^{3}$, Rita \\ C.P. Krumenauer ${ }^{3}$, Vinicius T. Richter ${ }^{4}$
}

\section{Clinical and endoscopic findings in children with stridor}

Palavras-chave: estridor, fibronasolaringobroncoscopia, anomalias congênitas da laringe.

Key-words: stridor, fibronasolaryngobroncoscopy, congenital anomalies of the larynx.

\section{Resumo / Summary}

\section{I} ntrodução: As anomalias da via aérea freqüentemente se apresentam com estridor. A sua principal causa em recémnascidos e lactentes são as alterações congênitas da laringe. O diagnóstico etiológico específico pode ser realizado com a fibronasolaringobroncoscopia, a qual é fundamental para o manejo adequado dessas crianças. Forma de estudo: prospectivo clínico de delineamento transversal de análise descritiva. Objetivo: Descrever as principais causas e achados clínicos associados ao estridor das crianças que se apresentam em nosso hospital. Material e Método: Realizouse um estudo prospectivo, no período de março de 2000 a abril de 2001, que avaliou todas as crianças encaminhadas ao Serviço de Otorrinolaringologia Pediátrica do Hospital da Criança Santo Antônio, cujo critério de inclusão foi a presença de estridor. Os pacientes foram então avaliados segundo protocolo definido a priori, que incluía, além de perguntas sobre história, exame físico e comorbidades associadas, o exame fibronasolaringobroncoscópico padronizado. Resultados: Foram avaliadas 125 crianças com estridor: 69 masculinos e 56 femininos. A idade média de apresentação foi de 19 meses. A maior parte dos pacientes avaliados veio da unidade de tratamento intensivo. Foram encontradas comorbidades em quase todos os casos, sendo a intubação prolongada a mais comum $(n=36,28,8 \%)$. Os diagnósticos mais freqüentes foram laringomalácia em 51 (40,8\%) pacientes e estenose subglótica em 22 (17,6\%). Conclusões: Mesmo que se tenha avaliado pacientes mais selecionados do que as grandes séries mundiais, as anomalias congênitas da laringe também foram a maior causa de estridor em nossa casuística. Em alguns casos com história de intubação prolongada foi difícil diferenciar a estenose subglótica congênita da adquirida. A presença de comorbidades é freqüente e pode auxiliar na seleção dos pacientes que necessitam de exame endoscópico.

\begin{abstract}
ntroduction: Abnormalities of the airway often present with stridor. The main cause in neonates and infants are the congenital defects of the larynx. The specific etiologic diagnose can be just obtained through fibronasolaryngobroncoscopy, which is necessary for the correct management of this children. Study design: Clinical prospective and descriptive cross-sectional study. Aim: To describe the main causes and associated clinical findings of stridor in children presenting to our hospital. Material and Method: A prospective investigation was performed from March 2000 to April 2001 to study all children evaluated at the Department of Pediatric Othorrinolaryngology of Hospital da Criança Santo Antônio with the inclusion criterion of stridor. These patients were examined according to a priori defined protocol, which includes besides questions about history, physical examination and associated comorbities, a standard fibronasolaryngobroncoscopy examination. Results: A group of 125 children ( 69 male and 56 female) were evaluated. The mean age at inclusion was 19 months. Most patients came from intensive care unit. Almost all cases had comorbidities, long-term endotracheal intubation been the most common finding. The most frequent diagnose were laryngomalacia $(n=51,40.8 \%)$ and subglottic stenosis $(n=22$, 17.6\%). Conclusions: Even in the presence of a more select sample than the great international series, we also found congenital anomalies of the larynx as the main cause of stridor. It was difficult to differentiate between congenital and acquired subglottic stenosis in some cases with a history of endotracheal intubation. The presence of comorbidities is frequent and may help to select the patients who will need endoscopic evaluation.
\end{abstract}

\footnotetext{
${ }^{1}$ Professor Adjunto da Disciplina de Otorrinolaringologia da Fundação Faculdade Federal de Ciências Médicas de Porto Alegre (FFCMPA) e Coordenador do Serviço de Otorrinolaringologia Pediátrica do Hospital da Criança Santo Antônio (HCSA) do Complexo Hospitalar Santa Casa de Porto Alegre (CHSCPA). Professor Titular da Disciplina de Pediatria da FFFCMPA.

${ }^{3}$ Médicos Residentes do Serviço de Otorrinolaringologia do CHSCPA. ${ }^{4}$ Acadêmico do 6o ano da FFFCMPA.

Trabalho realizado no Serviço de Otorrinolaringologia Pediátrica do HCSA do CHSCPA e Disciplina de Otorrinolaringologia da FFFCMPA. Endereço para correspondência: José Faibes Lubianca Neto - Rua Mostardeiro 157, sala 608 - 90430-001 - Porto Alegre RS - Brasil - E-mail: jlubianca@terra.com.br Trabalho Apresentado no II Congresso Triológico de Otorrinolaringologia de Goiânia, 2001.

Projeto Financiado pela Fundação de Amparo à Pesquisa do Estado do Rio Grande do Sul (FAPERGS) e pelo Programa CNPq PIBIC/2000. Artigo recebido em 31 de janeiro de 2002. Artigo aceito em 28 de março de 2002.
} 


\section{INTRODUÇÃO}

O estridor é um sintoma e não um diagnóstico, sendo a característica mais predominante da obstrução da via aérea na criança ${ }^{1}$. É gerado pela turbulência do ar durante a passagem por um sítio parcialmente obstruído. O estridor pode ser inspiratório, expiratório ou bifásico, conforme a localização e o tipo de alteração na árvore respiratória. Em lactentes, as principais causas são as alterações laríngeas. Destas, destacam-se a laringomalácia e a estenose subglótica² .

O termo laringomalácia foi introduzido em $1942^{3}$ e descrevia o colapso das estruturas supra-glóticas durante a inspiração. Anteriormente, as doenças congênitas da laringe que geravam estridor eram descritas conjuntamente como "estridor laríngeo congênito" . Apesar da laringomalácia ser a patologia de base na maioria dos recém-nascidos e lactentes que apresentam estridor ${ }^{5}$, não se pode desconsiderar a presença de outras causas ${ }^{6}$. Em 5 a 37\% dos casos se pode identificar lesão secundária de via aérea, somente diagnosticável através da fibronasolaringobroncoscopia?.

A estenose subglótica pode ser congênita ou adquirida. A congênita está ligada a uma falha na recanalização do lúmen laríngeo durante o envolvimento embrionário ${ }^{2}$. A forma adquirida mais comumente associase com intubação endotraqueal, mas também existem casos secundários à ingestão cáustica, complicações cirúrgicas, doenças granulomatosas e tumorais, entre outras. Nem sempre é fácil diferenciar entre a forma adquirida e a congênita, pois algumas vezes não se tem a oportunidade de avaliar o estridor antes do manuseio da via aérea por outros profissionais para controle da disfunção respiratória aguda, como em casos de intubação endotraqueal, cricotireoidotomia ou traqueostomia.

Apesar da laringomalácia e da estenose subglótica serem as causas mais freqüentes de estridor nos lactentes, existem outras alterações das vias aéreas que causam estridor e não devem ser tratadas com base num diagnóstico presuntivo ou inferencial ${ }^{1}$. O objetivo desse estudo é descrever as principais causas e comorbidades associados ao estridor nas crianças atendidas em nosso hospital.

\section{MATERIAL E MÉTODO}

Foi realizado um estudo transversal de planejamento prospectivo, no período de março de 2000 e abril de 2001, envolvendo 125 crianças. Foram incluídos no protocolo todos os pacientes encaminhados ao Serviço de Otorrinolaringologia Pediátrica do Hospital da Criança Santo Antônio (HCSA) do Complexo Hospitalar Santa Casa de Porto Alegre (CHSCPA) por apresentarem estridor. Praticamente metade dos pacientes (48\%) veio da Unidade de Tratamento Intensivo do HCSA. Dos 125 pacientes avaliados, 69 (55,2\%) eram do sexo masculino e $56(44,8 \%)$ do sexo feminino. A idade média de apresentação foi de 19 meses, variando entre 4 dias e 14 anos.

Os pacientes foram analisados segundo um protocolo previamente estabelecido pelos autores e aprovado pelo comitê de ética do CHSCPA, o qual incluía perguntas sobre idade, sexo, história médica atual e pregressa, exame físico e comorbidades associadas, além de exame fibronasolaringobroncoscópico padronizado. O exame fibronasolaringobroncoscópico incluiu a avaliação das fossas nasais, rinofaringe, orofaringe, hipofaringe, laringe, traquéia e brônquios fonte direito e esquerdo. O exame endoscópico foi realizado pelo autor sênior. O protocolo foi preenchido pelo mesmo profissional que realizou o exame, gerando um banco de dados digitado no Excell (Microsoft $\circledast$ ). As análises realizadas foram todas descritivas, em percentuais.

Planejou-se uma análise secundária sobre as duas causas mais comuns de estridor nas grandes séries mundiais: a laringomalácia e a estenose subglótica. A laringomalácia foi classificada em 5 tipos - Tipo I: prolapso de cartilagens cuneiformes aumentadas; Tipo II: exagero do formato ômega da epiglote que é longa e tubular e se curva sobre si mesma na inspiração; Tipo III: colapso anterior e medial das aritenóides na inspiração; Tipo IV: projeção posterior da epiglote na inspiração e Tipo V: prega ariepiglótica curta. A estenose subglótica também foi dividida conforme seu percentual de obstrução em 4 tipos: tipo I ( $25 \%$ de obstrução); tipo II (entre 51 e 70\%); tipo III (entre 71 e 90\%) e tipo IV (>90\%).

\section{RESULTADOS}

$\mathrm{Na}$ avaliação clínica, as principais comorbidades encontradas foram: intubação prolongada em 36 casos (28,8\%), refluxo gastro-esofágico em $23(18,4 \%)$ e malformações congênitas em 19 (15,2\%) (Gráfico 1), sendo 13 crianças $(10,4 \%)$ prematuras.

Dentre as malformações congênitas destacaram-se as malformações cardíacas em 9 casos $(7,2 \%)$ e malformações do tubo neural em 3 casos (2,4\%) (Gráfico 2).

Entre as regiões da via aérea, foi na laringe onde se encontrou o maior número de alterações (Tabela 1). A laringomalácia esteve presente em 51 pacientes $(40,8 \%)$, estenose subglótica em 22 (17,6\%), edema de prega vocal (PV) em 12 (9,6\%), granuloma de PV em 5 pacientes (4\%), paralisia de PV em $8(6,4 \%)$ e membrana laríngea anterior em 1 caso (0,8\%). A Tabela II compara os dados aqui obtidos com os de Holinger ${ }^{1}$ em lactentes abaixo de 30 meses. Descontando a diferença no tamanho entre as duas amostras, os percentuais são muito próximos (Tabela II).

Em nossa série de casos, houve 15 (29,4\%) casos de laringomalácia de tipo I, $4(7,8 \%)$ de tipo II, $4(7,8 \%)$ de tipo III, $11(21,5 \%)$ de tipo IV e $11(21,5 \%)$ de tipo V. Seis $(11,7 \%)$ casos não foram classificados. Ainda, aproxima 


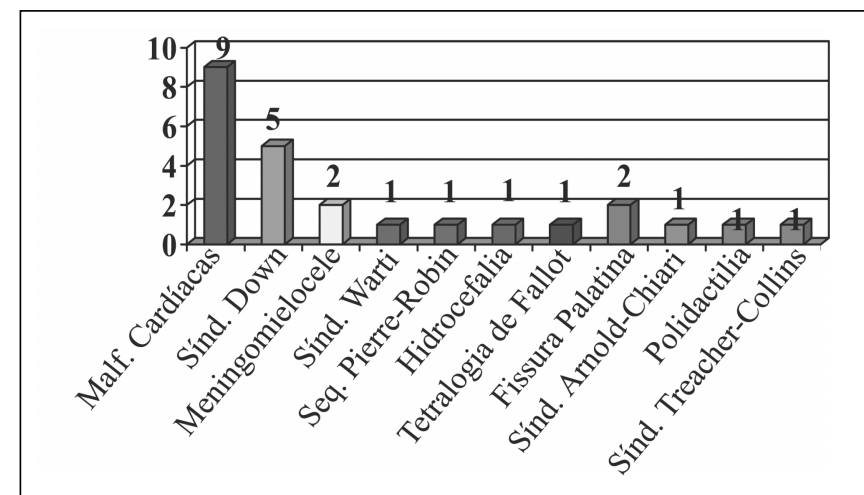

Gráfico 1. Co-morbidades Associadas ao Estridor.

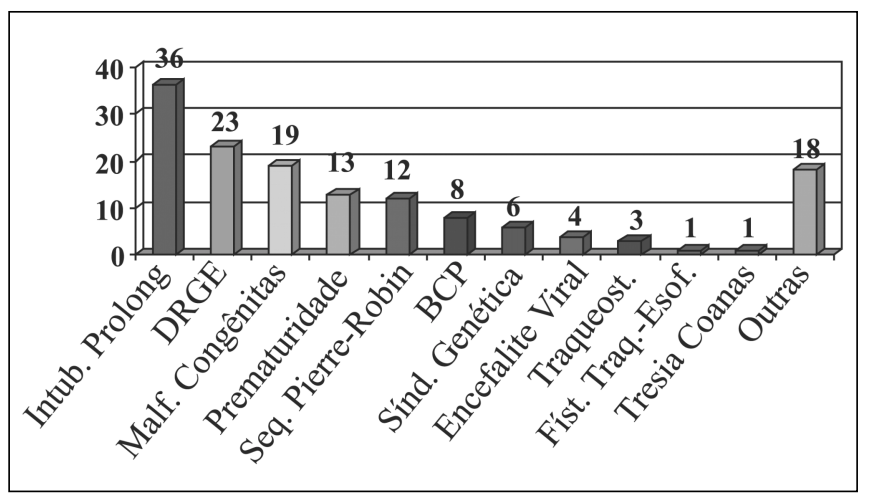

Gráfico 2. Malformações Congênitas e Síndromes Genéticas.

damente, 32\% dos pacientes apresentaram estenose subglótica tipo I, $45 \%$ tipo II, $9 \%$ tipo III e $14 \%$ tipo IV. O exame da traquéia revelou-se normal em 89 casos $(71,2 \%)$. Em 33 casos $(26,4 \%)$ a traquéia esteve alterada e não foi examinada em outros 3. A principal alteração detectada na traquéia foi a traqueomalácia, presente em 22 pacientes $(17,6 \%)$. O exame da carina e brônquios principais esteve normal em 115 casos e não foi realizado em 10 casos.

\section{DISCUSSÃO}

As anomalias congênitas das vias aéreas (VA) representam a maior causa de estridor em recém-nascidos e lactentes ${ }^{1,6,8}$. No entanto, a maioria destas crianças pode não apresentar estridor desde o nascimento. Quando presente nessa ocasião, geralmente as causas são obstruções fixas, como membranas laríngeas e estenose subglótica congênita. Freqüentemente o estridor aparece lentamente ou como períodos de exacerbação (alimentação, choro, decúbito), produzindo dispnéia, cianose ou apnéia ${ }^{9}$. Em nossa série de casos, a idade de apresentação variou de 4 dias até 14 anos (nesse extremo de idade está representado um paciente com paralisia cerebral e traqueostomizado, com granulações traqueais peritraqueostoma obstrutivas), indicando como pode ser ampla a faixa etária de percepção da sintomatologia, embora a média tenha sido ao redor dos dezenove meses de idade. Houve, também, uma leve predominância do sexo masculino. Dados similares foram encontrados em séries de pacientes com laringomalácia ${ }^{10} \mathrm{e}$ apnéia do sono ${ }^{11}$. Entretanto, a predominância do sexo masculino, em casos de anomalias congênitas da traquéia, estenose subglótica congênita e adquirida, pode sugerir que a população masculina tenha um risco maior de lesões obstrutivas da via aérea ${ }^{1}$.

A avaliação dos pacientes com estridor requer um detalhamento completo do sintoma, como data de início, característica, intensidade, fatores agravantes e de alívio e complicações. O estridor inspiratório é característico de lesões acima da caixa torácica. Este é gerado pelo colapso das estruturas laríngeas, conseqüente à pressão negativa criada na caixa torácica pelo movimento de inspiração. A lesão da estenose subglótica, mesmo sendo extra-torácica, produz estridor bifásico, por não ter sua morfologia modificada pelas pressões da via aérea. A traqueomalácia produz estridor predominantemente expiratório, como a asma. Exceção deve ser feita se houver comprometimento da traquéia intra e extra-torácica, onde teremos estridor bifásico.

No entanto, a caracterização simples do sintoma não é suficiente para o diagnóstico preciso. Holinger ${ }^{1}$ relatou uma série de 219 pacientes, onde houve 58 diagnósticos errôneos, corrigidos pela fibronasolaringobroncoscopia. Os principais diagnósticos errôneos foram asma, crupe e bronquiolite. Por isso o exame endoscópico é indispensável, uma vez que determina a causa exata do sintoma, além de excluir a concomitância de outras lesões na via aérea. Em um outro estudo ${ }^{8}, 37 \%$ dos pacientes tiveram mais de uma lesão na VA. Nenhum outro exame, como fluoroscopia, esofagograma baritado ou Rx lateral de pescoço pode ser tão definitivo e esclarecedor como a endoscopia nas lesões de via aérea ${ }^{12}$.

O exame endoscópico revelou alterações na maioria dos nossos casos. As principais alterações foram as da laringe, havendo uma predominância de laringomalácia e estenose subglótica. As pregas vocais foram normais em 86 dos casos $(68,8 \%)$. O exame também revelou epiglote em ômega em 22 casos. A epiglote ômega, isoladamente, não explica o estridor ${ }^{13}$, mas pode ser um fator contribuinte para o surgimento do sintoma, quando associada a outras alterações ${ }^{6}$. A traqueomalácia foi a principal alteração encontrada na traquéia, o que condiz com dados de outros pesquisadores.

A laringomalácia foi a anomalia congênita da laringe mais comum. Geralmente é uma condição auto-limitada, mas pode produzir episódios graves de apnéia, cor pulmonale e deficiências de desenvolvimento ${ }^{14}$. Sua origem está ligada a imaturidade neurológica da laringe. As pregas ariepiglóticas podem ser curtas, a epiglote em ômega e as cartilagens cuneiformes podem ser exageradamente grandes. Essas condições favorecem o prolapso da laringe durante a inspiração. A sintomatologia, na laringomalácia, é caracterizada por estridor, que inicia nas primeiras duas semanas de vida, 
Tabela 1. Principais Alterações da Laringe e Traquéia que Causam Estridor.

\begin{tabular}{lccc}
\hline $\begin{array}{l}\text { Alteração } \\
\text { (\%) }\end{array}$ & Lubianca et al. (\%)Holinger (7) & (\%)Altman (2) \\
\hline Laringomalácia & 40,8 & 59,8 & 68,4 \\
Estenose Subglótica & 17,6 & 20,5 & 6,9 \\
Traqueomalácia & 17,6 & 45,7 & 24,1 \\
Comp. Artéria Inominada & 1,6 & 34,3 & 5,7 \\
\hline
\end{tabular}

Tabela 2. Principais Alterações de Laringe que causam Estridor (< 30 meses).

\begin{tabular}{lcc}
\hline $\begin{array}{l}\text { Alteração } \\
(7)^{\star} \text {. (\%) }\end{array}$ & Lubianca et al.* (\%) & Holinger et al \\
\hline Laringomalácia & 58 & 60 \\
Estenose Subglótica & 19 & 20 \\
Paralisia de Prega Vocal & 12 & 13 \\
Outras & 11 & 7 \\
Total & 100 & 100 \\
\hline
\end{tabular}

* valores arredondados.

ocorrendo em repouso e/ou agitação. O estridor também é relacionado à posição da criança, sendo agravado pela posição supina e aliviado pela pronação. O comprometimento respiratório na laringomalácia geralmente não é severo e a criança não apresentará cianose e dispnéia. Mais freqüentemente se observam dificuldades para alimentação. O curso clínico é benigno na maioria dos pacientes, e a resolução dos sintomas ocorre até os 18 meses de idade 2 . Entretanto, em alguns casos, podem haver complicações respiratórias e ser necessária a supraglotoplastia ${ }^{14,15}$.

A estenose subglótica (ES) foi a segunda lesão mais freqüente encontrada em nossos casos. A ES é definida como uma medida menor que $4 \mathrm{~mm}$ do lúmen na região da cartilagem cricóide, em crianças a termo ${ }^{8}$. As falhas incompletas na recanalização da laringe na vida embrionária determinam os diversos tipos de ES. A sintomatologia pode não se manifestar até que haja alguma situação desencadeante. A maioria dos casos surge após infecções do trato respiratório, mas também após períodos de intubação prolongada. A doença do refluxo gastro-esofágico (DRGE), também está associada ao desenvolvimento de $\mathrm{ES}^{16}$. Halstead ${ }^{17}$ recentemente realizou um estudo comprovando que a DRGE tem um papel causal no desenvolvimento da ES. A mesma autora verificou que o tratamento clínico com drogas anti-refluxo pode resolver a sintomatologia em um terço dos casos. O tratamento da ES pode ser expectante nos casos menos severos, pois a maioria dos pacientes resolve os sintomas com o crescimento. Entretanto, alguns pacientes requerem tratamento cirúrgico, sendo a dilatação com balão ou com dilatadores específicos, o uso de laser de dióxido de carbono e a reconstrução laringotraqueal as principais opções.

As anomalias congênitas ou adquiridas da laringe comumente são acompanhadas de comorbidades. A intubação prolongada e as malformações congênitas foram exemplos bem representativos nos casos estudados. Dentre as malformações, as cardíacas foram as mais representadas na amostra, o que condiz com casuística de outros autores ${ }^{8}$. As infecções do trato respiratório superior são importantes comorbidades, pois desencadeiam a sintomatologia em muitos casos $^{2}$. No entanto, a grande maioria desses casos é tratada pelos pediatras na emergência ou na UTI de nosso hospital, não necessitando de nossa atuação. Por fim, a prematuridade pode estar envolvida na gênese de algumas causas de estridor, uma vez que constitui um fator determinante de imaturidade neurológica da árvore respiratória ${ }^{8}$ e esteve presente em muitos pacientes analisados.

Algumas limitações potenciais de nosso estudo devem ser ressaltadas. Como o estudo teve um enfoque diagnóstico, não se detalhou quais as medidas terapêuticas adotadas nos casos e se optou por não discuti-las. Em relação à amostra, nossas crianças representam uma população bem diferente da ambulatorial, na qual eventualmente o otorrinolaringologista pode se deparar com um caso de estridor. Grande parte dos pacientes foi oriunda da UTI do hospital, representando casos extremos de estridor. Mesmo assim, as causas de estridor não variaram muito em relação a outras casuísticas (vide Tabelas 1 e 2). A amostra altamente selecionada que se analisou explica a inexistência de casos de estridor de causa infecciosa, cardíaca, entre outras, que são mais comuns em amostras mais representativas da população ambulatorial.

\section{CONCLUSÃO}

Mesmo que se tenha avaliado pacientes mais selecionados do que as grandes séries mundiais, as anormalidades da laringe, congênitas ou não (laringomalácia e estenose subglótica), também foram a maior causa de estridor em nossa casuística. Em alguns casos com história de intubação prolongada foi difícil diferenciar a estenose subglótica congênita da adquirida. A presença de comorbidades foi freqüente e é um dos fatores que pode auxiliar na seleção dos pacientes que necessitam de exame endoscópico.

\section{REFERÊNCIAS BIBLIOGRÁFICAS}

1. Holinger LD. Etiology of Stridor in the Neonate, Infant and Child. Ann Otol Rhinol Laryngol 1980;89:397-400.

2. Cotton RT, Prescott CAJ. Congenital Anomalies of the Larynx. In: Practical Pediatric Otolaryngology. $1^{\text {st }}$ ed. Philadelphia: LippincottRaven Publishers; 1999.

3. Jackson C, Jackson CL. Diseases and Injuries of the Larynx. $1^{\text {st }}$ ed New York: Macmillan, 1942.

4. Sutherland GA, Lock HL. Congenital Laringeal Obstruction. Lancet 1897;2:653-655.

5. Mancuso RF, Choi SS, Zalzal ZH, Grundfast KM. Laryngomalacia. Arch Otolaryngol Head Neck Surg 1996;122:302-306.

6. Holinger LD, Konior RJ. Surgical Maneagement of Severe Laryngomalacia. Laryngoscope 1989;99:136-142. 
7. Albert D, Leighton S. Stridor and Airway Management. In: Cummings CW, Fredrickson JM, Harker LA, Krause CJ, Schuller DE, Richardson MA. Pediatric Otolaryngology Head \& Neck Surgery. $3^{\text {rd }}$ ed. St. Louis: Mosby; 1998. pp. 285-302.

8. Altman KW, Wetmore RF, Marsh RR. Congenital Airway Abnormalities in Patients Requiring Hospitalization. Arch Otolaryngol Head Neck Surg 1999; 125:525-528.

9. Richardson MA, Cotton RT. Anatomic abnormalities of the pediatric airway. Ped Clin of North Am 1984;31:821-834.

10. Holinger PH, Johnston KC, Schiller F. Congenital Anomalies of the Larynx. Ann Otol Rhinol Laryngol 1954;63:581-606.

11. Guilleminault C, van den Hoed J, Mitler MM. Clinical overview of the sleep apneas syndromes. In: Sleep Apnea Syndromes. New York: Allan R. List Inc; 1978.

12. Tunkel DE, Zalzal GH. Stridor in infants and children: ambulatory evaluation and operative diagnosis. Clin Pediatr (Phila.) 1992;31:3855.
13. Belmont JR, Grundfast K. Congenital Laryngeal Stridor (Laryngomalacia): Etiologic Factors and Associated Disorders. Ann Otol Rhinol Laryngol 1984;93:430-437.

14. Zalzal GH, Anon JB, Cotton RT. Ann Otol Rhinol Laryngol 1987;96:72-76.

15. Roger G, Denoyelle F, Triglia JM, Garabedian EM. Severe Laryngomalacia: Surgical indications and results in 115 patients. Laryngoscope 1995;105:1111-1117.

16. Yellon RF. The Spectrum of Reflux-Associated Otolaryngologic Problems in Infants and Children. Am J Med 1997;103(5A): 125129.

17. Halstead LA. Gastroesophageal reflux: A critical factor in pediatric subglottic stenosis. Otolaryngol Head Neck Surg 1999;120:683688. 